\title{
ESTRATEGIAS DE TRÁNSITO DE ADOLESCENTES CENTROAMERICANOS INDEPENDIENTES: ENFRENTANDO LA FRONTERA VERTICAL EN MÉXICO
}

Aída Silva Hernández ${ }^{1}$

\begin{abstract}
¿De qué manera enfrentan el tránsito por México los adolescentes centroamericanos que migran de manera independiente?, ¿cómo experimentan el contexto de frontera vertical en el territorio? En el presente artículo el concepto de frontera vertical pretende englobar una doble expresión empírica: la que corresponde al Estado mexicano, que dispone un sistema de control inmigratorio a lo largo del país, y la frontera vertical extraoficial, caracterizada por una serie de riesgos y arbitrariedades cometidas por diversos actores en contra de los inmigrantes. Desde la perspectiva de la agencia y a través de estudios de caso de adolescentes guatemaltecos y salvadoreños se revelan las estrategias de tránsito que buscan hacer frente a los obstáculos potenciados por dichas expresiones de frontera.
\end{abstract}

Palabras clave: frontera vertical, migración adolescente independiente, tránsito, migración centroamericana.

\section{Introducción}

El presente trabajo analiza la situación de México como país de tránsito de adolescentes centroamericanos hacia Estados Unidos con el fin de describir y analizar las estrategias implementadas por estos sujetos para hacer frente a los obstáculos que se interponen en su movilidad en un contexto de frontera vertical. El tránsito se entiende como una condición liminal del inmigrante en términos físicos, sociales y estructurales producto de la escasez de recursos económicos y sociales con los que cuenta, por su internación indocumentada a México y el consecuente desplazamiento clandestino, y debido a la existencia de una atmósfera periférica al ocultamiento caracterizada por una serie de riesgos y arbitrariedades cometidas por diversos actores en contra de los inmigrantes.

1 Profesora en la Facultad de Humanidades de la Universidad Autónoma de Baja California Campus Tijuana, México. 
Se recurre al concepto de frontera vertical como una propuesta para aprehender ese contexto liminal en su conjunto. Por un lado, la frontera vertical de Estado, que hace referencia al aparato de control inmigratorio implementado a lo largo del país a través de operativos, retenes y detenciones, extendiendo así las características otrora exclusivas de las fronteras geopolíticas como espacios de ejercicio de poder y como filtros selectivos de la migración. Por otro lado, una frontera vertical extraoficial que se superpone a la primera, obstaculizando el tránsito de manera tácita a través de grupos delictivos que se sustentan en la explotación de la persona inmigrante, no sólo en términos económicos y laborales, sino corporales. Se argumenta que ambos sistemas restrictivos del tránsito migratorio, si bien de distinta naturaleza, se articulan y se potencian como factores de riesgo, originando que los inmigrantes adolescentes desarrollen estrategias de tránsito que apuntan a evadir y/o afrontar los obstáculos de estas fronteras verticales de manera simultánea e indistinta.

Utilizando estudios de caso de adolescentes guatemaltecos y salvadoreños que se desplazaron de forma independiente a la tutoría adulta hasta llegar a la frontera norte de México se recuperan las experiencias de tránsito por el país, examinando en particular el encuentro con esas diversas manifestaciones de frontera vertical y las estrategias de tránsito implementadas. Las experiencias muestran cómo las fronteras verticales atraviesan las dimensiones personales, grupales y estructurales con una carga de factores económicos y socio-culturales que de alguna manera legitiman ese contexto, al grado que el abuso se convierte en algo sistemático.

En el primer apartado se hace una revisión de los conceptos centrales de la problemática seguido por el recuento de los flujos de menores de edad centroamericanos que se desplazaron por México de manera independiente en los últimos años. Continúa el apartado que consiga la experiencia de tránsito de cinco centroamericanos, cerrando con una serie de reflexiones finales.

\section{Tránsito, frontera vertical y estrategia adolescente. Precisiones conceptuales}

El tránsito se concibe regularmente como una cuestión lineal: la fase del proceso migratorio entre la salida del lugar de origen y el establecimiento en el lugar de destino. Esa fase se ubicada espacialmente en los llamados países de tránsito. Sin embargo, autores como Düvell, Schapendonk, Collyer, de Haas y Hess, cuestionan la clasificación de una nación como "país de tránsito" por considerar que cuenta con una carga política que resulta conveniente para los países dominantes (de destino pretendido), los cuales, en función de sus intereses, externalizan sus políticas de control migratorio hacia los países geográficamente adyacentes o cercanos, atribuyendo la problemática migratoria propia a esos "países de tránsito". En esa 
lógica, se les adjudica a los "países de tránsito" el cometido de "controlar" el flujo de personas que cruza de manera irregular sus fronteras geopolíticas².

La situación geográfica de México no sólo lo distingue como "país vecino" de Estados Unidos sino como "país de tránsito" de centroamericanos, lo que ha venido a reconfigurar sus fronteras, sobre todo en las últimas tres décadas, para mostrar actualmente dos expresiones fronterizas: la geopolítica, que sirve para "puntualizar los límites del Estado-nación y que establece una demarcación político-territorial y cultural de gran envergadura, frontera que retiene y que se significa como punto de asimetría y riesgo" ${ }^{\prime 3}$.

Otra es la mencionada frontera vertical, en la que:

las acciones y operativos [de las autoridades migratorias] se han extendido a lo largo y ancho de su geografía, multiplicando las estaciones y estancias migratorias para controlar los movimientos y contener los flujos de personas que se internan en el país sin autorización, filtrando a la vez los desplazamientos hacia el vecino país del norte ${ }^{4}$.

Desde esa conceptualización de México como "país de tránsito", los indocumentados en el país devienen en "migrantes en tránsito": los que cruzan sin habitar, catalogados a priori con permanentes intenciones de paso.

De Genova considera que "la 'ilegalidad' migratoria es una condición de espacialidad social que con frecuencia constituye el centro de las formas particulares en que los migrantes son racializados como 'extranjeros ilegales' dentro de los límites de un estado-nación" ${ }^{5}$. La "ilegalidad" de la migración radica en "haber transgredido la autoridad soberana del Estado-nación" y que esa soberanía, "como instancia del desenfrenado autoritarismo en la vigilancia fronteriza, la detención, la deportación, y más, asume un carácter marcadamente absolutista" . En una tesis contundente, el autor explica que "la posibilidad de la deportación, y no la deportación en sí, es la que históricamente ha permitido que los trabajadores migrantes indocumentados sean considerados como una mercancía claramente desechable" ${ }^{\prime \prime}$. Esto se aplica en los países de destino, tanto como en el panorama mexicano como "país de tránsito".

A la latencia de ser expulsado le llama De Genova "deportabilidad" (deportability). Dicha posibilidad obliga al individuo a llevar a cabo acciones que

2 DÜVELL, Franck. Transit Migration: A Blurred and Politicised Concept, p. 416. En esta y en todas las citas sucesivas de fuentes en inglés, la traducción es mía.

3 KEARNEY, Michael. Fronteras fragmentadas, fronteras reforzadas, p. 559 y 561.

4 ANGUIANO, Eugenia; TREJO, Alma. Políticas de seguridad fronteriza y nuevas rutas de movilidad de migrantes mexicanos y guatemaltecos, p. 50 y 51.

5 DE GENOVA, Nicolas. Migrant "Illegality" and Deportability in Everyday Life, p. 439.

6 IDEM. The Legal Production of Mexican/migrant 'Illegality', p. 175.

7 IDEM, Migrant "Illegality"..., op. cit., p. 438. 
buscan solventar la situación: ocultándose y moviéndose en transportes y zonas marginales donde la vulnerabilidad y la exposición a riesgos es una constante. Con la frontera vertical la eventualidad de la repatriación se encuentra vigente a lo largo del territorio.

A esto se suma en México, más claramente en la última década, la presencia de grupos delictivos especializados en atacar y robar a inmigrantes indocumentados en sus rutas habituales, principalmente las anexas al tren de carga conocido como "La Bestia", transporte masivo de centroamericanos hacia Estados Unidos ${ }^{8}$. Entre los principales rasgos que caracterizan la violencia contra los migrantes indocumentados en México se encuentra "la percepción de que se trata del grupo social subordinado que mejor empata con las características de vulnerabilidad, en combinación con la impunidad que suele obtener el perpetrador ante prácticas corruptas del personal de inmigración" ${ }^{\prime \prime}$.

De acuerdo con Jácome, esta situación se enmarca en una violencia estructural que involucra factores sociales, económicos y políticos provenientes de al menos ocho fuerzas complementarias e interrelacionadas:

Las prácticas migratorias mexicanas; las actitudes históricas en México relativas a la migración; la xenofobia contra los transmigrantes; la influencia de Estados Unidos sobre la política migratoria de México; el marco jurídico mexicano; la creciente presencia del tráfico de drogas; la pobreza, la marginación y las estructuras de la violencia en las poblaciones locales a lo largo de la ruta; $y$ las condiciones socio-económicas en los países de origen ${ }^{10}$.

Es así como se genera en México el entorno amplio de las fronteras verticales, un campo que va más allá de la sola disposición de políticas de control inmigratorio.

El migrante indocumentado "en tránsito" queda fijo entonces en un lugar social de "derechos estratificados, una forma muy jerarquiza de ciudadanía flexible como producto que corresponde a esta racionalidad espacial del neoliberalismo que caracteriza a los países de destino"11. Con ello, el tránsito se convierte en una situación que algunos autores califican de "reajuste en una trayectoria fragmentada"12; de espera, como una fase de aparente inmovilidad dentro de un proceso de movilidad ${ }^{13}$, o de "formas suspendidas de existencia"14.

${ }_{8}$ CASILLAS, Rodolfo. The Dark Side of globalized Migration: The Rise and Peak of Criminal Networks. The Case of Central Americans in Mexico, p. 300.

9 CASILLAS, op. cit., p. 302 y 304.

${ }^{10}$ JÁCOME, Felipe. Trans-Mexican Migration: a Case of Structural Violence, p. 26.

${ }^{11}$ HESS, Sabine. De-naturalising Transit Migration. Theory and Methods of An Ethnographic Regime Analysis, p. 436.

${ }^{12}$ COLLYER, Michael; DE HAAS, Hein. Developing Dynamic. Categorisations of Transit Migration, p. 478.

${ }^{13}$ SCHAPENDONK, Joris. Migrants' im/mobilities on their way to the EU: Lost in transit? p. 578.

14 HESS, op. cit., p. 435. 
Ese estado de suspensión social, incluso, es asumido por los propios migrantes como parte de lo que implica su travesía hacia el norte.

El escenario social señalado concierne a los migrantes que presentan la característica común de encontrarse en algún tipo de nivel de pobreza, si bien sus experiencias de tránsito liminal se diferencian en función de la edad, el género, el acompañamiento, las capacidades agenciales y los motivos de traslado. Distinguimos en este universo a nuestros sujetos de estudio, individuos originarios de países centroamericanos que se encuentran en edades de la adolescencia ${ }^{15}$ y que se desplazan sin la compañía de un tutor adulto, es decir, de manera independiente. Efectúan un desplazamiento indocumentado bajo una normatividad primordialmente vigilante, ya sea por parte de las autoridades migratorias o de instancias gubernamentales de asistencia al menor de edad "no acompañado", las que comúnmente, en el afán de "proteger", detienen y controlan la trayectoria migratoria de los adolescentes.

La movilidad de los adolescentes centroamericanos se caracteriza entonces por la implementación de una serie de mecanismos de afrontamiento que denominamos estrategias de tránsito.

Conceptualmente se podrían señalar los antecedentes de las estrategias de tránsito en los componentes que integran las estrategias de afrontamiento (coping strategies), y que se refieren a "acciones y actividades que tienen lugar dentro de estructuras existentes"16. Las estrategias de afrontamiento conllevan por definición una situación de riesgo y una condición de vulnerabilidad. En la definición de Ruiz, el riesgo en la migración es considerado un proceso. Es "estar expuesto en el camino a una cosa o a una persona que es potencialmente una amenaza o un peligro, a tal grado que pueda perjudicar o dañar, a veces irreparablemente, el proyecto de migrar o la integridad física del migrantes"17. La vulnerabilidad, en este sentido, quedaría definida como una condición de susceptibilidad a "la exposición a una amenaza o un peligro identificado como tal por las personas"18. Específicamente, el riesgo en la migración de adolescentes se entiende "como la probabilidad de que ocurran determinados comportamientos sociales y prácticas institucionales que no toman en consideración, o incluso se aprovechan de la predisposición intrínseca de la niñez y la adolescencia, para exponerla en una situación de desprotección, descuido o abuso"19.

${ }^{15}$ Se retoma la definición etaria de adolescentes como "las personas entre los 12 años de edad cumplidos y los 18 años incumplidos". CONGRESO DE LA UNIÓN-MÉXICO, Art. 2.

${ }^{16}$ ERIKSEN, Siri; BROWN, Katrina; KELLY, Mick. The Dynamics of Vulnerability: Locating Coping Strategies in Kenya and Tanzania, p. 288.

${ }^{17}$ RUIZ, Olivia. Riesgo, migración y espacios fronterizos: una reflexión, p. 276.

18 Ibidem.

${ }^{19}$ ACUÑA, Guillermo. Migración y trabajo infantil y adolescente: una aproximación para la construcción de una agenda regional, p. 28. 
En las estrategias de afrontamiento la capacidad de respuesta a esas amenazas está conformada por la agencia y la resiliencia. La primera se define como la capacidad de una persona para manejar recursos a su favor o para evitar exponerse a daños potenciales; esa agencia personal está influenciada por la agencia de otros y por estructuras más amplias, como son las instituciones ${ }^{20}$. La resiliencia es el tipo de agencia que se da en condiciones de vulnerabilidad, definiéndose como "la capacidad humana para enfrentar, sobreponerse, ser fortalecido o incluso, ser transformado por las adversidades de la vida"21. La resiliencia "privilegia el enfoque en las fortalezas, no en el déficit o problema e involucra a los individuos, familias, grupos, comunidades e instituciones a que sean parte de la solución con el conjunto de recursos internos y externos que permitan enfrentar situaciones críticas de todo tipo"22.

A partir de lo anterior, se definen las estrategias de tránsito como el uso por parte del adolescente, entendido como agente, de los recursos personales, interpersonales e institucionales que es capaz de poner a su alcance, en una secuencia de decisiones y acciones que se dirigen a hacer frente a los obstáculos que se interponen a la consecución de sus objetivos migratorios (con resultados que no necesariamente corresponden a dichos fines). La movilidad que protagonizan implica una transgresión territorial, por lo que las estrategias de tránsito primordialmente buscan disminuir, evitar o superar los obstáculos que provienen de esa condición migratoria indocumentada. El doble contexto de frontera vertical los involucra así de manera directa.

\section{Adolescente, independiente, centroamericano e indocumentado: aproximación a las dimensiones de la problemática en México ${ }^{23}$}

La emigración de centroamericanos a Estados Unidos en volúmenes significativos empezó a destacar en la década de los ochenta, principalmente desde Guatemala, Honduras, El Salvador y Nicaragua. Entre 1995 y 2003 el paso de centroamericanos por México registró un volumen que fluctuaba entre 201 mil y 265 mil eventos. En 2004 el volumen se incrementó a 324 mil eventos para alcanzar un máximo de 433 mil en 2005. A partir de 2006 la tendencia se dio a la baja debido principalmente a las medidas de control inmigratorio de

\footnotetext{
${ }^{20}$ SUMNER, Andy; MALLETT, Rich. Snakes and Ladders, buffers and passports: rethinking poverty, vulnerability and wellbeing, p. 20.

${ }^{21}$ GROTBERG, Edith. A Guide to Promoting Resilience in Children: Strengthening the Human Spirit, p. 9.

22 QUINTERO, Ángela. Resiliencia: contexto no clínico para trabajo social, p. 7.

${ }^{23}$ El paso de centroamericanos por México, dado el carácter irregular del ingreso, sólo se maneja en términos de estimaciones partiendo de estadísticas de centroamericanos deportados por las autoridades mexicanas y estadounidenses, y por el monto de centroamericanos radicados en la Unión Americana.
} 
México y de Estados Unidos, a la disminución de la demanda laboral producto de la crisis en Estados Unidos y a las condiciones de inseguridad imperantes en el tránsito por México ${ }^{24}$. A partir de 2009 se observó una estabilización en el orden de los 130 mil eventos, con un repunte en 2012 de 183 mil eventos de tránsito irregular de centroamericanos por México ${ }^{25}$.

En cuanto al flujo específico de niños y adolescentes centroamericanos independientes en tránsito por México (referidos en las fuentes estadísticas como "no acompañados"), las aproximaciones a su volumen se basan en el monto de menores de edad repatriados a sus países de origen por el Instituto Nacional de Migración (INM), por el número de niños y adolescentes centroamericanos que ingresa a la Red de Albergues del Sistema Nacional para el Desarrollo Integral de la Familia (SN-DIF), y por el volumen de aprehensiones de este grupo poblacional realizadas por el aparato de control inmigratorio de Estados Unidos. Las dos fuentes mexicanas coinciden en señalar como patrones del flujo de menores de edad centroamericanos independientes que ocho de cada diez son varones entre 13 y 17 años de edad, con una escolaridad promedio de segundo básico, equivalente a nivel primaria en México.

Gráfica 1. Eventos de retorno asistido de menores de edad centroamericanos "no acompañados" por país de origen, 2007-2014

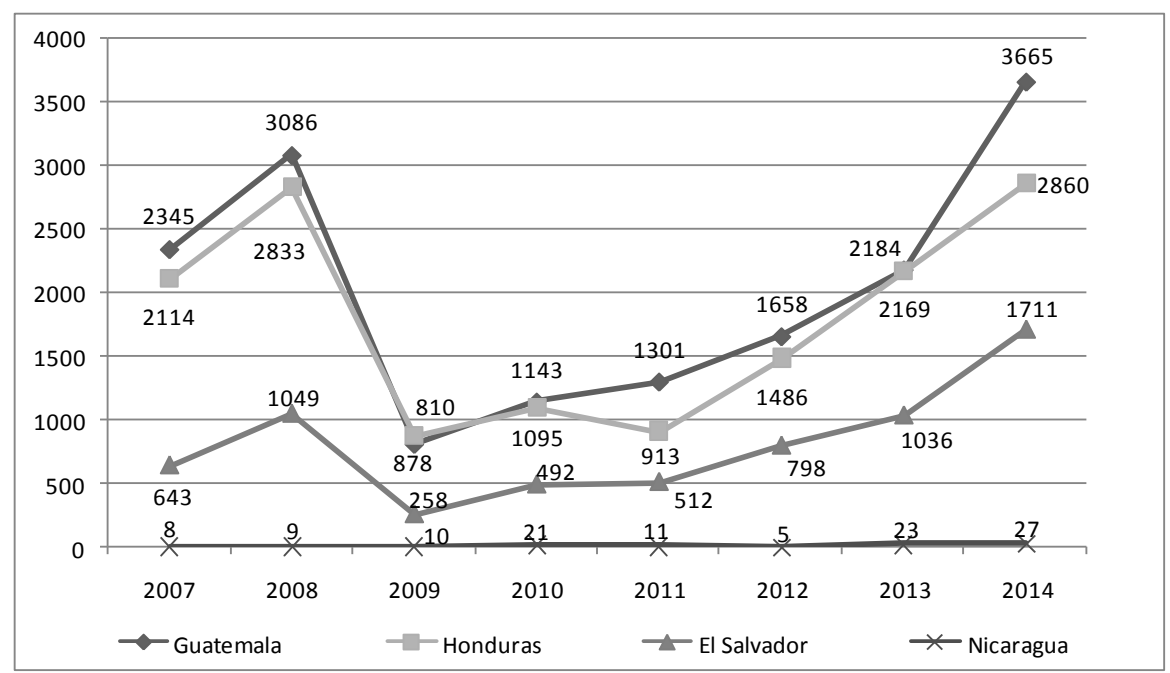

Fuente: Elaboración propia con datos de los Boletines mensuales de Estadística Migratoria 2008-2015, Instituto Nacional de Migración.

${ }^{24}$ RODRÍGUEZ, Ernesto; BERÚMEN, Salvador; RAMOS, Luis. Migración centroamericana de tránsito irregular por México. Estimaciones y características generales, p. 2.

25 ITAM. Migración centroamericana en tránsito por México hacia Estados Unidos: diagnóstico y recomendaciones. Hacia una visión integral, regional y de responsabilidad compartida, p. 12. 
Entre los centroamericanos "no acompañados" expulsados por autoridades mexicanas destacan los originarios de Guatemala, Honduras y El Salvador, como lo muestra la Gráfica 1. De 2008 a 2009 se observó una drástica reducción en el número de eventos de "retorno asistido" de menores de edad, seguido por un crecimiento constante y significativo de 2011 a 2014, periodo en el que pasó de un total de 2.737 eventos de niños y adolescentes independientes repatriados, a 8.263 en este último año.

En territorio mexicano funciona la llamada Red de Albergues integrada por instituciones gubernamentales y de la sociedad civil dedicados a la tención de niños y adolescentes migrantes, nacionales y extranjeros, en condición de "no acompañados". En dicha Red, coordinada por el Sistema Nacional DIF, se ha venido registrando un incremento constante de centroamericanos que recurren en su tránsito a los servicios de los albergues (Cuadro 1). De 2009 a 2010 se dio el incremento más significativo, cuando los usuarios centroamericanos se sextuplicaron, pasando de 515 a 3.229 ingresos, la mayoría en albergues de la frontera sur de México. A partir de 2010 el creciente desplazamiento por México de centroamericanos menores de edad "no acompañados" derivó en que se integraran a la Red albergues no-fronterizos, ubicados en Oaxaca y Veracruz, los cuales registran un incremento explosivo en la atención a niños y adolescentes centroamericanos, como se muestra en este mismo cuadro.

\section{Cuadro 1. Extranjeros atendidos en la red de albergues} del Sistema Nacional DIF, 2001-20013

\begin{tabular}{|l|c|c|c|c|c|c|c|c|c|c|c|c|c|}
\hline $\begin{array}{l}\text { Ubicación } \\
\text { del albergue }\end{array}$ & 2001 & 2002 & 2003 & 2004 & 2005 & 2006 & 2007 & 2008 & 2009 & 2010 & 2011 & 2012 & 2013 \\
\hline $\begin{array}{l}\text { Frontera } \\
\text { norte }\end{array}$ & 150 & 53 & 131 & 161 & 309 & 410 & 425 & 365 & 249 & 173 & 228 & 347 & 681 \\
\hline Frontera sur & - & - & - & - & 77 & 371 & 488 & 467 & 266 & 3002 & 3013 & 338 & 4906 \\
\hline $\begin{array}{l}\text { Intermedios } \\
\text { (Oaxaca y } \\
\text { Veracruz) }\end{array}$ & - & - & - & - & - & & & & & 54 & 235 & 521 & 1957 \\
\hline Totales & 150 & 53 & 131 & 161 & 386 & 781 & 913 & 832 & 515 & 3229 & 3476 & 4206 & 7544 \\
\hline
\end{tabular}

Fonte: elaboración propia con dados de los Anuarios estadísticos del Sistema Nacional DIF, 2008-2014 y Ortega, 2009. (-) No había(n) albergue(s) dentro de la red del Sistema Nacional DIF.

Una tercera referencia que permite aproximarse al volumen de menores de edad independientes que se desplazan por México proviene del Departamento de Seguridad Nacional de Estados Unidos a través de su oficina de la Patrulla Fronteriza, encargada de aprehender a los migrantes indocumentados en el cruce de la frontera México-Estados Unidos. De acuerdo con esta fuente, en el año fiscal de 2012 lograron ingresar al país vía México un total de 10.146 niños y adolescentes de origen centroamericano, monto que se duplicó en 2013 con 20.805 detenciones 
y en 2014 alcanzó un máximo histórico de 51.705 detenciones ${ }^{26}$. Las principales razones de los menores de edad independientes para internarse de manera indocumentada a ese país son "escapar de la violencia, del abuso o la persecución en sus países de origen; reunirse con algún familiar en la Unión Americana; buscar trabajo para mantenerse a sí mismos, a sus familias de origen o a sus familias de formación, o que fueron llevados a Estados Unidos por redes de trata"27.

En conjunto, las estadísticas más recientes aportadas por las tres fuentes refieren montos del tránsito de menores de edad centroamericanos independientes que han venido en aumento, y que a pesar del mayor número de detenciones y de devoluciones desde México, la entrada a Estados Unidos ha llegado a volúmenes sin precedentes. Dicho comportamiento sugiere que este grupo de población migrante es de tal dimensión que el poder selectivo de la frontera vertical no alcanza a escindir, o bien, que se relaja para "dejar pasar". En cualquier caso los adolescentes centroamericanos independientes entran en la espiral de condiciones liminales y peligrosas del tránsito.

Las experiencias que se examinan a continuación señalan el complejo entramado de componentes estructurales que trazan el estadio de tránsito por México en los últimos años, así como la capacidad de agencia de estos sujetos para articular estrategias a su paso.

\section{Estrategias de tránsito de los adolescentes centroamericanos para afrontar la frontera vertical}

Para este trabajo se recurre a estudios de caso construidos a partir de entrevistas en profundidad de corte biográfico: cinco adolescentes centroamericanos que fueron contactados en albergues de Tijuana, Baja California, cuando habían experimentado el tránsito por México en una ocasión o más, realizando su desplazamiento entre 2010 y 2013 de manera independiente a la tutoría adulta y en forma voluntaria. Dos chicos habían cumplido 18 años de edad al momento de la entrevista pero habían recorrido el país siendo menores de edad, por lo que estuvieron sujetos a la normatividad inmigratoria bajo esa calidad legal, al igual que el resto de los casos.

Se trata de dos grupos de hermanos que se acompañaron en el trayecto: tres de Ciudad de Guatemala y dos de Santa Ana, El Salvador, todos con ingreso irregular a México, viviendo la repatriación como una amenaza constante. El nivel escolar general es de primaria, ya sea inconclusa o terminada. Entre los casos la experiencia laboral es mínima o nula. Provienen de entornos urbanos donde predominaba la violencia sobre la pobreza como factor que impulsó la

\footnotetext{
${ }^{26}$ HOMELAND SECURITY, 2015.

${ }^{27}$ OFFICE OF REFUGEE RESETTLEMENT, 2014.
} 
emigración, proceso caracterizado por la precariedad y por escasas redes sociales de apoyo.

Los hermanos guatemaltecos son Eleazar de 18 años de edad, Yolanda de 17 y Bruno de 15; los hermanos salvadoreños son Liliana de 18 y Simón de 16 años $^{28}$. Cuando fueron entrevistados, tanto Eleazar como los hermanos salvadoreños contaban con el status migratorio de refugiado en México.

Se presentan las experiencias de tránsito siguiendo el orden del proceso migratorio: desde el cruce de la frontera sur de México, el encuentro con las fronteras verticales y su llegada a la frontera geopolítica México-Estados Unidos.

\section{"Preparación" para la salida del país de origen y el cruce de la frontera sur de México}

Los cinco adolescentes carecían de información y de contactos útiles para su desplazamiento en México. Desde antes de salir de sus países sabían que el escaso dinero con el que contaban sería insuficiente para completar su trayecto, previendo solventar su trayectoria con estancias temporales en lugares donde pudieran conseguir algún trabajo o acercándose a fuentes de recursos como las iglesias católicas o cristianas y mediante la solicitud de dádivas o limosna. "No conocíamos más ni cómo cruzar a México; pensábamos: 'a ver si encontramos un trabajo', y quedarnos por ahí o algo... cómo fuera"29.

La preocupación por los riesgos en la migración no se expresa como un factor que se revise detenidamente. Los riesgos se conocen y se asumen, pues no hay presupuesto para optar por vías más seguras en el tránsito. Estos costos finalmente se evalúan como menores frente a la conveniencia de salir. La compañía en la salida se da con los hermanos como parte de una maniobra de protección, tutoría temprana y de sinergia.

El cruce de la frontera sur de México se percibe como un umbral de poca dificultad en cuanto a la vigilancia migratoria pero complicado por su geografía (el cruce del río, montañas y selva) y por los peligros que representan los grupos delictivos en la zona. Atravesar el Suchiate toma "cinco minutos, pasamos y $\mathrm{ya}^{\prime \prime 30}$. Una vez en territorio mexicano los adolescentes asumen su situación migratoria irregular y la problemática de desplazarse como menores de edad sin acompañamiento de alguna persona adulta, por lo que actúan en consecuencia, es decir, ocultándose.

Pronto entran en contacto con agentes del Instituto Nacional de Migración, entre ellos algunos que desarrollan prácticas corruptas en detrimento de los más

\footnotetext{
${ }^{28}$ Todos los nombres son seudónimos. Edades al momento de la entrevista.

${ }^{29}$ Entrevista realizada a Bruno. Tijuana, 2012.

${ }^{30}$ Entrevista realizada a Eleazar. Tijuana, 2012.
} 
vulnerables: "ahí [en Chiapas] hacían la ley del que tiene dinero lo dejan ir y al que no, lo deportan"31.

La frontera sur de México, principalmente en los límites con el estado de Chiapas, destaca como una zona de conexión entre el ejercicio de control de las políticas inmigratorias mexicanas, la penetración de grupos delictivos que abusan de los migrantes y la circulación continua de migrantes en condiciones de pobreza, combinación que cristaliza en el establecimiento de redes delictivas que tienen a los sujetos migrantes como "mercancía" en el sentido del potencial de explotación que guarda el migrante mismo, de su cuerpo, sus recursos y su trabajo. Conforme el adolescente se aleja de la frontera geopolítica la explotación perdura aunque se expresa de maneras distintas.

\section{Estrategias en el tránsito}

Los estudios de caso se caracterizaron por presentar un tránsito de duración considerable: Eleazar por ejemplo, recorrió el país en cuatro ocasiones entre 2010 y 2013, obligado por deportaciones desde México o Estados Unidos o por regreso voluntario a Guatemala. Por su parte, los hermanos salvadoreños duraron ocho meses en tránsito para poder llegar a la frontera norte de México.

El tránsito se muestra gradual y toma diversas trayectorias en función de los recursos disponibles: cuando faltan recursos monetarios hay que detenerse a conseguirlos; cuando se encuentran recursos institucionales como los albergues, hay que detenerse a aprovecharlos.

Destacan dos situaciones en esta fase de la movilidad: la individual, que hace referencia a las condiciones de pobreza en las que se realiza el tránsito, a las condiciones de incertidumbre y angustia que viven los chicos y a la posición de resistencia asumida por el adolescente frente a los riesgos. Otras son las condiciones de carácter estructural que sitúan y retienen a estos adolescentes pobres en continuas situaciones de riesgo y en la clandestinidad, de tal manera que el tránsito significa encontrarse en "formas de existencia pendientes, suspendidas, o dicho de otra manera, en formas de asentamiento precarias y provisionales"32. Así lo narra Eleazar: "Es parte del camino que lo asalten a uno, lo golpeen, las hambres, el caminar. Uno ya sabe a lo que viene, ya sabe qué riesgo va a correr uno y si pasa-pasa, y si no, igual, ya es una mentalidad que traen todos. Ahí sí que es suerte de cada quien"33.

Como respuesta a la desprotección en su tránsito por México, los adolescentes subrayan sus capacidades de agencia y resiliencia y crean o

\footnotetext{
31 Ibidem.

32 HESS, op. cit., p. 435.

${ }^{33}$ Entrevista realizada a Eleazar. Tijuana, 2013.
} 
potencian fuentes de recursos a través de relaciones de apoyo emergentes, en su mayoría esporádicas pero esenciales para la satisfacción de sus necesidades inmediatas de alimentación, dinero, ropa y alojamiento. Los apoyos provienen de iniciativas particulares: adultos que comúnmente responden favorablemente a las solicitudes de ayuda de los adolescentes gracias a que éstos han desarrollado aptitudes para el convencimiento.

Sin embargo, el encuentro con personas que ofrecen ayuda es azaroso por la diversidad de intereses de por medio: puede tratarse de un apoyo honesto o uno que busque atentar contra su integridad física. La salvadoreña Liliana comentó la situación que le tocó vivir en los albergues:

Había otro salvadoreño también, como que secuestraba gente [...] que la llevaba engañada. Nosotros por la forma de hablar no confiamos en ese hombre y nosotros le contamos a otro señor y dijo: 'Sí, no digan nada pero ese hombre no es buena espina; ni vayan a pensar irse con él. Si ustedes quieren venirse conmigo, vénganse ${ }^{34}$.

Asimismo, los adolescentes se relacionan con diversas instituciones que encuentran a su paso, como albergues o comedores de la sociedad civil o iglesias. Parte de sus estrategias de tránsito consiste en incorporar a estas instituciones como recursos, cuidando que no se interpongan o coarten su "libertad", es decir, que les impidan tomar decisiones autónomas. Este factor de autonomía se defiende constantemente e incluso prefieren moverse de manera independiente aun cuando les represente mayores dificultades para su subsistencia. En el tránsito se alterna el contacto con dependencias dedicadas a la vigilancia inmigratoria (INM) y las dedicadas a su protección (DIF). Desde la percepción de los adolescentes entrevistados ambas dependencias coinciden en la finalidad de retener y de controlar su desplazamiento, por lo que son precavidos de relacionarse con DIF y en evitar al Instituto Nacional de Migración. Yolanda cuenta el encuentro con personal de DIF en un parque del estado de Jalisco: "Una señora [de DIF] nos quería meter al DIF; decía que estaba muy peligroso, que por qué íbamos a venir hasta Tijuana. Nosotros le dijimos que a trabajar y dijo que no, que mejor en el DIF. Le dijimos que no y nos tuvimos que ir [del lugar donde estaba la persona por temor a ser retenidos]"35. Por esa percepción de vigilancia y control, los adolescentes centroamericanos muestran preferencia por apoyos de organizaciones de la sociedad civil, visualizándolos como instituciones libres de intereses gubernamentales.

La movilidad independiente de los adolescentes implica un itinerario extenso por la precariedad ya señalada. El transporte más utilizado en el tránsito a

\footnotetext{
${ }^{34}$ Entrevista realizada a Liliana. Tijuana, 2012.

${ }^{35}$ Entrevista realizada a Yolanda. Los Ángeles, Ca., 2013.
} 
partir de Arriaga, Chiapas, es el tren de carga conocido como "La Bestia", medio que en sí mismo representa un serio peligro de accidentes, además que alrededor del tren subsiste una estructura delictiva orientada a explorar de distintas maneras a los migrantes (secuestro, extorsión, violaciones sexuales, robo a mano armada, explotación y muerte). "Se suben y uno se tiene que esconder porque a veces uno no tiene nada [que robarle] y al ver ellos que uno no tiene nada lo botan [del tren] o lo machetean, les daban por aquí [en el cuello] y los dejaban tirados"36. El recorrido en "La Bestia" se realiza por tramos de uno o dos días.

En un esfuerzo por contrarrestar los riesgos, y ante el débil ejercicio de la normatividad del Estado, los migrantes que viajan en La Bestia establecen mecanismos de sobrevivencia a través de lo que se pudieran llamar "comunidades de protección", es decir, grupos de personas que regularmente comparten la misma nacionalidad, que transmiten información y efectúan acciones dirigidas a elevar su seguridad (rutas a evitar, actitudes convenientes, identificación de delincuentes, aprendizaje de reconocimiento de códigos del peligro, por ejemplo). A estas "comunidades" se adhieren los menores de edad, tanto varones como mujeres:

Un señor y otros amigos nos apoyaban; eran de Honduras. Nos ayudaban a subirnos al tren, nos ayudaban con las mochilas. Ellos venían cuidando que nadie me hiciera daño porque sólo yo venía de mujer. Porque ahí se subían asaltantes y se subían a violar a las muchachas delante de todos. Yo así, me agaché, bien agachada y bien encogidita, yo escondidita por cualquier cosa, pero gracias a Dios, no pasó nada ${ }^{37}$.

Esta es una de las mayores preocupaciones de los hermanos varones: proteger la integridad física de sus hermanas, quienes se saben altamente vulnerables a violaciones sexuales, si bien los hombres no están exentos de esta vejación. "No agarramos el tren. Lo íbamos a agarrar pero luego no porque mi hermano [Eleazar] dijo que tenía que pagar para que me subiera al tren, pa' que me metieran ahí donde van los costales de no sé qué, de comida. Mi hermano pequeño [Bruno] dijo que no, que no subiéramos al tren porque me iban a violar"38.

El raite o aventón en camiones de carga es el transporte opcional para avanzar hacia el norte, con el riesgo que implica salirse de las rutas de tránsito públicas, ya que el conductor puede resultar un agresor.

De acuerdo con una clasificación migratoria, en las rutas de los centroamericanos por México se distinguen tres tipos de sitios: las plataformas, que son zonas de concentración donde arriban, se reagrupan, resguardan y parten los grupos de migrantes; los puertos de enlace o nodos, es decir, lugares

\footnotetext{
${ }^{36}$ Entrevista realizada a Eleazar. Tijuana, 2012.

37 Entrevista realizada a Liliana. Tijuana, 2012.

38 Entrevista realizada a Yolanda. Los Ángeles, Ca., 2013.
} 
provistos de rutas alternas donde se re-direccionan los migrantes, y tercero, los llamados sitios de paso, en los que no se detiene el migrante o sólo hace una escala breve $^{39}$. En los casos de los adolescentes que aquí se presentan, la frontera sur funcionó como plataforma mientras que Oaxaca y el Distrito Federal como nodos; el resto del trayecto quedó sujeto a las eventualidades. La ruta implicó tiempos de espera y de reajuste y se fue definiendo sobre la marcha a partir de la información transmitida por otros migrantes y bajo el criterio básico de buscar la mayor seguridad posible, aún a costa de grandes rodeos.

Por otro lado, se advierte que cuando los adolescentes son detectados y retenidos por el INM ven la posibilidad de evitar la repatriación solicitando el refugio ante la Comisión Mexicana de Ayuda a Refugiados (COMAR). Simón hace referencia a la manera que fueron conducidos al INM: arbitrariamente, el personal del DIF los presentó/entregó a estas autoridades.

Nos fuimos a buscar un albergue y llegamos al albergue del DIF pero nos mandaron a migración [INM] y en migración estuve dos meses. Nos dijeron que si nos daban el refugio y todo eso, que ya podíamos estar legales en México; lo único que [nos pidieron fue que] firmáramos un papel en el que decía que si salíamos del país, el gobierno de México ya no se hacía responsable de nosotros, que era responsabilidad de nosotros si ya faltábamos a un peligro o algo asi ${ }^{40}$.

Este recurso administrativo es un giro trascendental por el tiempo y la forma en que los adolescentes ven detenida su movilidad. Durante el proceso de solicitud de refugio en el Distrito Federal, Liliana, Simón, Eleazar y Yolanda (quien se desistió y fue repatriada a su país), permanecieron recluidos en estaciones migratorias entre dos y cinco meses, lo que resultó en desesperación y sentido de pérdida sobre el control de la trayectoria. El refugio para los adolescentes significa la posibilidad de moverse con mayor seguridad por territorio mexicano ya que desaparece la necesidad de esconderse, sin que por ello se perciba como un mecanismo para el asentamiento. Por lo mismo, aún con el status migratorio de refugiados no se asumen con el derecho de recibir algún tipo de asistencia institucional o protección social, pues en algunos casos no fue ofrecida y en otros fue rechazada por el adolescente al considerarla inadecuada para sus planes.

De tal manera, son múltiples las experiencias en el tránsito por México que van llevando al adolescente a confeccionar mecanismos de afrontamiento. Como sus acciones están en función de los obstáculos más que de los recursos, el semblante que retratan los adolescentes de sí mismos se caracteriza por la seguridad en sus decisiones, la evaluación de sus habilidades y capacidades y la

\footnotetext{
${ }^{39}$ CASILLAS, Rodolfo. Las rutas de los centroamericanos por México, un ejercicio de caracterización, actores principales y complejidades, p. 166.

${ }^{40}$ Entrevista realizada a Simón. Tijuana, 2012.
} 
convicción de saber cuidarse solos. Con ello, la construcción del ser-adolescente podría parecer épica. Sin embargo, también muestran que el factor etario fluctúa entre la ponderación de sus capacidades y el reconocimiento de sentirse personas aún en formación, de ahí que fácilmente tengan expresiones tanto de fortalezas como de vulnerabilidad: "se puede decir que estamos pequeños; aunque ya soy mayor [de edad], me siento como una niña pequeñita"41.

\section{La frontera México-Estados Unidos}

Similar a lo que sucede en frontera sur, en la frontera norte de México se recalcan las carencias de los adolescentes. Al llegar, las tensiones del recorrido los obliga a tomar un tiempo para recuperarse y descansar en los albergues. "Es una pesadilla, y lo que le espera todavía a uno, porque esto es sólo la mitad"42.

Entonces encuentran una frontera geopolítica que por primera vez en su largo tránsito parece un tope prácticamente infranqueable por el aparato de control fronterizo estadounidense. Más allá de las dificultades físicas que advierten los chicos, la frontera norte de México se patentiza como filtro selectivo de las migraciones. Los filtros para los adolescentes no sólo están dados por las políticas de control fronterizo de la Unión Americana sino por su nacionalidad centroamericana misma, su minoría de edad, su condición de "no acompañados", sus condiciones de pobreza, su falta de capacitación laboral, el escaso manejo del inglés y su débil red social.

Los adolescentes centroamericanos sin experiencia previa de internación indocumentada a Estados Unidos pretenden repetir la estrategia de movilidad desarrollada en México, sin considerar las diferencias socioeconómicas y culturales entre ambos países. Básicamente hay una falta de planeación y de conocimiento de la forma de internarse a la Unión Americana y de las acciones posteriores a realizar en caso de lograr cruzar, por lo que las probabilidades de aprehensión son altas.

Por esto y por temor a sufrir agresiones en el cruce, los hermanos salvadoreños decidieron no cruzar y permanecer en México mientras fraguaban algún tipo de estrategia alterna. En cuanto a los hermanos guatemaltecos, fueron aprehendidos por la Oficina de Aduanas y Protección Fronteriza de los Estados Unidos. Yolanda y Bruno quedaron internados en albergues para menores de edad en tanto se revisaba la posibilidad de otorgarles asilo en Estados Unidos, mientras que Eleazar, siendo ya mayor de edad (2013), fue detenido durante nueves meses en una cárcel del Servicio de Inmigración y Control de Aduanas de Estados Unidos antes de ser deportado a su país.

\footnotetext{
${ }^{41}$ Entrevista realizada a Liliana. Tijuana, 2012.

${ }^{42}$ Entrevista realizada a Eleazar. Tijuana, 2012.
} 


\section{Reflexiones finales}

Los estudios de caso aportan información respecto a la manera que los adolescentes centroamericanos adoptan una serie de medidas estratégicas para hacer frente al contexto de frontera vertical oficial y extraoficial que encuentran en México.

El carácter irregular de su inmigración los hace moverse en formas que exhiben su vulnerabilidad, como sucede en ese "escenario" que representa "La Bestia", en los albergues como nodos, la transportación de "aventón" y la solicitud de limosna en las calles. La vulnerabilidad queda asociada a las repercusiones de un tejido social fraccionado entre simpatizantes y agresores de los inmigrantes centroamericanos y a las políticas inmigratorias de Estado, incluyendo sus serias deficiencias en la procuración de seguridad pública.

Aún con el apoyo que los adolescentes centroamericanos logran obtener de dependencias gubernamentales, desde su experiencia la distinción entre las instituciones encargadas de vigilar y de proteger se diluye. Para ellos finalmente, son autoridades que por diversas razones pretenden conducir su movilidad.

Los adolescentes constatan la frontera sur de México como una frontera porosa para el ingreso pero selectiva dentro de la clandestinidad, ya que es preciso contar con un mínimo de recursos monetarios para "pagar" por entrar y por el "permiso" de seguir en tránsito, cuotas que van para los grupos criminales y para las autoridades corruptas. Por otro lado, la frontera norte se alza como un muro sin concesiones a su minoría de edad, a su pobreza y a la falta de documentos. Intentar el cruce a Estados Unidos, permanecer en la frontera o regresar a algún punto del trayecto es una decisión que los desconcierta.

Esto es: la magnitud de la exclusión social en la que se encuentran se magnifica de manera contundente en el contexto de frontera vertical en México, ejercicio de poder que distingue a los que "habrán de pasar" de los rechazados, facilitando en el intermedio, la proliferación de riesgos y abuso sistemático.

\section{Bibliografía}

ACUÑA, Guillermo. Migración y trabajo infantil y adolescente: Una aproximación para la construcción de una agenda regional. Conferencia presentada en el Tercer Foro de Organizaciones No Gubernamentales de Iberoamérica. 3, 4 y 5 de octubre de 2006, Uruguay, Marcha Global contra el Trabajo Infantil/OIT.

ANGUIANO, Eugenia; TREJO, Alma. Políticas de seguridad fronteriza y nuevas rutas de movilidad de migrantes mexicanos y guatemaltecos. Liminar, v. V, n. 2, 2007, p. 47-65.

CASILLAS, Rodolfo. The Dark Side of globalized Migration: The Rise and Peak of Criminal Networks. The Case of Central Americans in Mexico. Globalizations, v. 8, n. 3, 2011, p. 295-310. 
CASILLAS, Rodolfo. Las rutas de los centroamericanos por México, un ejercicio de caracterización, actores principales y complejidades. Migración y desarrollo, v. 10, 2008, p. 157-174.

COLLYER, Michael; DE HAAS, Hein. Developing Dynamic. Categorisations of Transit Migration. Population, Space and Place, n. 18, 2012, p. 468-481.

CONGRESO DE LA UNIÓN-MÉXICO. Ley para la Protección de los Derechos de las Niñas, Niños y Adolescentes 2000. México, 2000. Disponible en: <http:// www.salud.gob.mx/unidades/ cdi/nom/compi/L290 500.html>. Consultado: 07.07.2010.

DE GENOVA, Nicholas. The Legal Production of Mexican/migrant "Illegality". Latino Studies, n. 2, 2004, p. 160-185.

DE GENOVA, Nicholas. Migrant "Illegality" and Deportability in Everyday Life. Annual Review Anthropology, v. 31, 2002, p. 419-447.

DÜVELL, Franck. Transit Migration: A Blurred and Politicised Concept. Population, Space and Place, n. 18, 2012, p. 415-427.

ERIKSEN, Siri; BROWN, Katrina; KELLY, Mick. The Dynamics of Vulnerability: Locating Coping Strategies in Kenya and Tanzania. The Geographical Journal, v. 171, n. 4, 2005, p. 287-305.

GROTBERG, Edith. A Guide to Promoting Resilience in Children: Strengthening the Human Spirit. Early Childhood Development: Practice and Reflections, n. 8, 1995, p. 1-39.

HESS, Sabine. De-naturalising Transit Migration. Theory and Methods of An Ethnographic Regime Analysis. Population, Space and Place, n. 18, 2012, p. 428440.

HOMELAND SECURITY. Cuadro Unaccompanied Alien Children Encountered by Fiscal Years 2009-2014; Fiscal Year 2015 to date (October 1, 2014-December 31, 2014). Disponible en: <htpp://www.cbp.gov/newsroom/stats/southwest-borderunaccompanied-children>. Consultado: 05.01.2015.

INSTITUTO NACIONAL DE MIGRACIÓN. Boletín mensual de Estadísticas Migratorias 2014. México: SEGOB/INM, 2015.

INSTITUTO NACIONAL DE MIGRACIÓN. Boletín mensual de Estadísticas Migratorias 2013. México: SEGOB/INM, 2013 [sic].

INSTITUTO NACIONAL DE MIGRACIÓN. Boletín mensual de Estadísticas Migratorias 2012. México: SEGOB/INM, 2013.

INSTITUTO NACIONAL DE MIGRACIÓN. Boletín mensual de Estadísticas Migratorias 2011. México: SEGOB/INM, 2011 [sic].

INSTITUTO NACIONAL DE MIGRACIÓN. Boletín mensual de Estadísticas Migratorias 2010. México: SEGOB/INM, 2010 [sic].

INSTITUTO NACIONAL DE MIGRACIÓN. Boletín mensual de Estadísticas Migratorias 2009. México: SEGOB/INM, 2009 [sic].

INSTITUTO NACIONAL DE MIGRACIÓN. Boletín mensual de Estadísticas Migratorias 
2008. México: SEGOB/INM, 2009.

INSTITUTO NACIONAL DE MIGRACIÓN. Boletín mensual de Estadísticas Migratorias 2007. México: SEGOB/INM, 2008.

ITAM. Migración centroamericana en tránsito por México hacia Estados Unidos: diagnóstico y recomendaciones. Hacia una visión integral, regional y de responsabilidad compartida. México: ITAM, 2014.

JÁCOME, Felipe. Trans-Mexican Migration: a Case of Structural Violence. Working Paper Series Georgetown University, n. 2, 2008, p. 1-38.

KEARNEY, Michael. Fronteras fragmentadas, fronteras reforzadas. In MUMMERT, Gail (ed.). Fronteras fragmentadas, identidades múltiples. México: COLMICH/CIDEM, 1999, p. 559-571.

OFFICE OF REFUGEE RESETTLEMENT, 2014. Disponible en: <http://www.acf.hhs. gov/programs/orr/programms/ucs/about>. Consultado: 17.06.2014.

ORTEGA, Ricardo. Análisis de los Anuarios Estadísticos 2001-2007 de la red de albergues de tránsito de niñas, niños y adolescentes migrantes. México: DIF/ UNICEF, 2009.

QUINTERO, Ángela. Resiliencia: contexto no clínico para trabajo social. Revista Latinoamericana de Ciencias Sociales Niñez y Juventud, v. 3, n. 1, 2005, p. 73-94.

RODRÍGUEZ, Ernesto; BERÚMEN, Salvador; RAMOS, Luis. Migración centroamericana de tránsito irregular por México. Estimaciones y características generales. Apuntes sobre Migración, n. 1, 2011, p. 1-8.

RUIZ, Olivia. Riesgo, migración y espacios fronterizos: una reflexión. Estudios Demográficos y Urbanos, v. 16, n. 2, 2001, p. 257-284.

SCHAPENDONK, Joris. Migrants' im/mobilities on their way to the EU: Lost in transit? Tijdschrift voor Economische en Sociale Geografie, v. 103, n. 5, 2012, p. 577-583. SISTEMA NACIONAL PARA EL DESARROLLO INTEGRAL DE LA FAMILIA. Anuario estadístico 2013. Estrategia de prevención y atención a niñas, niños y adolescentes migrantes y repatriados no acompañados. México: SNDIF, 2014.

SISTEMA NACIONAL PARA EL DESARROLLO INTEGRAL DE LA FAMILIA. Anuario estadístico 2012. Estrategia de prevención y atención a niñas, niños y adolescentes migrantes y repatriados no acompañados. México: SNDIF, 2013.

SISTEMA NACIONAL PARA EL DESARROLLO INTEGRAL DE LA FAMILIA. Anuario estadístico 2011. Estrategia de prevención y atención a niñas, niños y adolescentes migrantes y repatriados no acompañados. México: SNDIF, 2012.

SISTEMA NACIONAL PARA EL DESARROLLO INTEGRAL DE LA FAMILIA. Anuario estadístico 2010. Estrategia de prevención y atención a niñas, niños y adolescentes migrantes y repatriados no acompañados. México: SNDIF, 2011.

SISTEMA NACIONAL PARA EL DESARROLLO INTEGRAL DE LA FAMILIA. Anuario estadístico 2009. Estrategia de prevención y atención a niñas, niños y adolescentes migrantes y repatriados no acompañados. México: SNDIF, 2010.

SISTEMA NACIONAL PARA EL DESARROLLO INTEGRAL DE LA FAMILIA. Anuario estadístico 2008. Estrategia de prevención y atención a niñas, niños y adolescentes 
migrantes y repatriados no acompañados. México: SNDIF, 2009.

SUMNER, Andy; MALLETT, Rich. Snakes and Ladders, buffers and passports: rethinking poverty, vulnerability and wellbeing. Working paper, n. 83, 2011, p. $1-37$.

\section{Abstract \\ Transit strategies of independent Central American adolescents migrants: coping with Vertical Border in Mexico}

How do independent Central American adolescents migrants face transit through Mexico? How do they experience the Mexican vertical border context? In this paper the concept of vertical border tries to gather a double empirical expression: the Mexican State one, which refers to the immigration enforcement system spread throughout the country, and an unofficial vertical boundary, characterized by a set of risks and abuses committed against immigrants by different actors. From the perspective of Agency, and based on case studies of Guatemalans and Salvadorans adolescents, this article reveals the transit strategies of this immigrants in their attempt to overcome the obstacles enhanced by such boundary expressions.

Keywords: vertical border, independent adolescent migration, transit, Central American migration.

Recebido para publicación en 17.03.2015

Aceptado para publicación en 14.05.2015

Received for publication on March, 17 $7^{\text {th }}, 2015$.

Accepted for publication on May, 14 $4^{\text {th }}, 2015$.

ISSN impresso: 1980-8585

ISSN eletrônico: 2237-9843

www. http://dx.doi.org/10.1590/1980-85852503880004407 